\title{
Time Domain Simulation of Harmonic Ultrasound Images and Beam Patterns in 3D Using the $k$-space Pseudospectral Method
}

\author{
Bradley E. Treeby ${ }^{1}$, Mustafa Tumen ${ }^{2}$, and B.T. Cox $^{2}$ \\ 1 College of Engineering and Computer Science, The Australian National University \\ 2 Department of Medical Physics and Bioengineering, University College London
}

\begin{abstract}
A $k$-space pseudospectral model is developed for the fast full-wave simulation of nonlinear ultrasound propagation through heterogeneous media. The model uses a novel equation of state to account for nonlinearity in addition to power law absorption. The spectral calculation of the spatial gradients enables a significant reduction in the number of required grid nodes compared to finite difference methods. The model is parallelized using a graphical processing unit (GPU) which allows the simulation of individual ultrasound scan lines using a $256 \times$ $256 \times 128$ voxel grid in less than five minutes. Several numerical examples are given, including the simulation of harmonic ultrasound images and beam patterns using a linear phased array transducer.
\end{abstract}

Keywords: ultrasound simulation, nonlinear, $k$-space methods, GPU.

\section{Introduction}

The simulation of ultrasound propagation through biological tissue has many applications. These include the design of ultrasound transducers; the development of new apodization, beamforming, and signal processing techniques; training ultrasonographers to use ultrasound equipment and interpret ultrasound images; medical image registration; and treatment planning [9. However, simulations based on the conservation equations that govern the propagation of acoustic waves in tissue can be very computationally expensive. This is because the size of the computational domains can equate to hundreds of wavelengths in each spatial dimension. Moreover, established numerical techniques such as the finite element and finite difference methods require on the order of 10 grid nodes per wavelength to achieve acceptable accuracy. This yields a requirement for computational domains with thousands of grid nodes in each spatial dimension. Consequently, 3D simulations can require large amounts of memory and take days or weeks to run, even when distributed computing systems are used [8]4.

To avoid directly solving the fundamental acoustic conservation equations (or the equivalent wave equations), researchers have previously been forced to make a number of simplifying assumptions. For example, for computing the shape of the acoustic field produced by different ultrasound transducers, a parabolic

G. Fichtinger, A. Martel, and T. Peters (Eds.): MICCAI 2011, Part I, LNCS 6891, pp. 363-370, 2011. (C) Springer-Verlag Berlin Heidelberg 2011 
Table 1. Required grid sizes and memory capabilities for ultrasound simulations in three-dimensions using a $k$-space pseudospectral model. The number of required elements is based on two grid nodes per wavelength using a sound speed of $1500 \mathrm{~m} / \mathrm{s}$. The required memory is based on the storage of 20 single-precision matrices (these are used to store the tissue properties, acoustic variables, etc).

\begin{tabular}{cccc}
\hline $\begin{array}{c}\text { Domain Size } \\
\left(\mathrm{cm}^{3}\right)\end{array}$ & $\begin{array}{c}\text { Maximum Freq } \\
(\mathrm{MHz})\end{array}$ & $\begin{array}{c}\text { Number of Required } \\
\text { Elements }\end{array}$ & $\begin{array}{c}\text { Required Memory } \\
(\mathrm{GB})\end{array}$ \\
\hline $5^{3}$ & 1 & $67^{3}$ & 0.022 \\
& 5 & $333^{3}$ & 2.8 \\
& 10 & $667^{3}$ & 22 \\
\hline $10^{3}$ & 1 & $133^{3}$ & 0.18 \\
& 5 & $667^{3}$ & 22 \\
& 10 & $1333^{3}$ & 180 \\
\hline
\end{tabular}

approximation is often used 2]. However, this approximation makes it difficult to accurately model the wave-field away from the transducer axis. For the simulation of diagnostic ultrasound images, both ray-tracing and convolution based approaches are frequently used 146 . However, these approaches are not normally extended to model multiple reflections or nonlinear effects. The latter is particularly important as modern ultrasound scanners rely on nonlinear wave propagation (in which acoustic waves at lower frequencies produce waves at higher frequency harmonics as they propagate) for tissue harmonic imaging which gives improved image clarity and contrast. There is thus a need for ultrasound simulation tools with less restrictive assumptions but improved computational efficiency.

Here, the $k$-space pseudospectral method is applied to the simulation of nonlinear ultrasound propagation in biological tissue. The spectral calculation of the spatial derivatives is performed using the fast Fourier transform (FFT) and only requires two grid nodes per acoustic wavelength to achieve acceptable accuracy [10. This significantly relaxes the requirement for dense computational domains compared to finite difference methods. The use of the FFT also provides a convenient method for parallelization using graphical processing units (GPUs). A summary of the required computing capabilities is given in Table 1, the equivalent finite difference model would require more than two orders of magnitude more elements rendering most of these problems intractable for normal computing systems. Note, for nonlinear simulations, the maximum frequency may be several times higher than the centre frequency of the transducer. In addition to the spectral calculation of spatial derivatives, the accuracy of the finite difference time step is also improved using a $k$-space adjustment (this makes the temporal discretization scheme exact in the case of linear propagation in homogeneous media [10]). For heterogeneous media, this is similar to using a pseudospectral method with a higher order scheme for the temporal derivative, but has a smaller memory penalty. This approach has previously been used to model linear wave propagation in biomedical photoacoustics [3] and ultrasonics [4]. 
The numerical solution is based directly on coupled acoustic conservation equations governing nonlinear wave propagation in heterogeneous media. A novel equation of state is used which accounts for material nonlinearity in addition to power law acoustic absorption (biological tissue has been experimentally shown to exhibit absorption characteristics of this form 9]). An accurate model for acoustic absorption is of particular importance for nonlinear wave propagation as the generation of higher frequencies via nonlinearity is delicately balanced with their absorption. Previous full-wave models have included only thermoviscous absorption (which is inaccurate for biological tissue) or a spectrum of relaxation processes [5. While the latter can account for power law absorption over a limited frequency range, this requires an a priori fitting of an array of relaxation times for each value of absorption and set of simulation parameters.

\section{Model Development}

The equations governing the nonlinear propagation of compressional acoustic waves in heterogeneous fluid media can be derived from the mass, momentum, and energy conservation laws for continuum mechanics. Under the assumption of a quiescent, isotropic, and inviscid medium in which shear waves can be neglected, the momentum and mass conservation equations can be respectively written as

$$
\rho_{0} \frac{\partial \mathbf{u}}{\partial t}+\nabla p=-\rho \frac{\partial \mathbf{u}}{\partial t}-\frac{1}{2} \rho_{0} \nabla \mathbf{u}^{2}, \quad \frac{\partial \rho}{\partial t}+\nabla \cdot\left(\rho_{0} \mathbf{u}\right)=-\nabla \cdot(\rho \mathbf{u}) .
$$

Here $p$ and $\rho$ are the acoustic pressure and density, $\mathbf{u}$ is the particle velocity where $\mathbf{u}^{2}=\mathbf{u} \cdot \mathbf{u}, \rho_{0}$ is the ambient (background) density, and only terms up to second order in the acoustic variables have been retained. Following the approach taken by Aanonsen et al. [1, the second order terms which appear on the right hand side in (11) can be re-written in terms of the Lagrangian density via the repeated substitution of the acoustic equations in linearized form. If only cumulative nonlinear effects are important (as is the case for biomedical ultrasound in the absence of microbubbles), the Lagrangian density terms can then be neglected which yields the expressions

$$
\frac{\partial \mathbf{u}}{\partial t}=-\frac{1}{\rho_{0}} \nabla p, \quad \frac{\partial \rho}{\partial t}=-\rho_{0} \nabla \cdot \mathbf{u}-\mathbf{u} \cdot \nabla \rho_{0}+\frac{1}{\rho_{0} c_{0}^{4}} \frac{\partial p^{2}}{\partial t} .
$$

The final term in the mass conservation equation corresponds to a convective nonlinearity in which the particle velocity contributes to the wave velocity.

Neglecting thermoviscous losses and instead including a phenomenological loss operator to account for arbitrary power law absorption [13, the expansion of the total pressure using a Taylor series about the equilibrium density for a heterogeneous medium yields the equation of state

$$
p=c_{0}^{2}\left(\rho+\mathbf{d} \cdot \nabla \rho_{0}+\frac{B}{2 A} \frac{\rho^{2}}{\rho_{0}}-\tau \frac{\partial}{\partial t}\left(-\nabla^{2}\right)^{\frac{y}{2}-1} \rho-\eta\left(-\nabla^{2}\right)^{\frac{y+1}{2}-1} \rho\right) .
$$


The five terms within the brackets separately account for linear wave propagation, heterogeneities in the ambient density, material nonlinearity, power law acoustic absorption, and sound speed dispersion. Here $c_{0}$ is the isentropic sound speed, $\mathbf{d}$ is the particle displacement vector, and $\tau$ and $\eta$ are the absorption and dispersion proportionality coefficients given by $\tau=-2 \alpha_{0} c_{0}^{y-1}$ and $\eta=$ $2 \alpha_{0} c_{0}^{y} \tan (\pi y / 2)$. These operators account for acoustic absorption of the form $\alpha=\alpha_{0} \omega^{y}$ where $\alpha_{0}$ is the absorption coefficient prefactor in $\mathrm{Np}(\mathrm{rad} / \mathrm{s})^{-y} \mathrm{~m}^{-1}$ and $y$ is the power law exponent 13 . Note, the $\nabla \rho_{0}$ terms in (2) and (3) cancel to first order and are not included in the discrete equations given below.

The three expressions given in (2) and (3) form a coupled set of equations for the acoustic particle velocity, density, and pressure. It is also possible to combine these expressions into a single second order wave equation for the acoustic pressure. Neglecting higher order absorption, nonlinearity, and heterogeneity terms, this can be written in the form of a modified Westervelt equation

$$
\nabla^{2} p-\frac{1}{c_{0}^{2}} \frac{\partial^{2} p}{\partial t^{2}}-\frac{1}{\rho_{0}} \nabla \rho_{0} \cdot \nabla p+\frac{\beta}{\rho_{0} c_{0}^{4}} \frac{\partial^{2} p^{2}}{\partial t^{2}}+\left(\tau \frac{\partial}{\partial t}\left(-\nabla^{2}\right)^{\frac{y}{2}}+\eta\left(-\nabla^{2}\right)^{\frac{y+1}{2}}\right) p=0
$$

where $\beta=1+B / 2 A$ is the coefficient of nonlinearity. An equation of this form without the power law absorption term has previously been used for the simulation of ultrasound images in 2D using the finite difference method [8]7.

Neglecting higher order absorption and nonlinearity effects, the conservation equations in (2) written in discrete form using a $k$-space pseudospectral method are given by

$$
\begin{aligned}
\frac{\partial}{\partial \xi} p^{n} & =\mathbb{F}^{-1}\left\{i k_{\xi} \kappa \mathbb{F}\left\{p^{n}\right\}\right\}, \\
u_{\xi}^{n+1} & =u_{\xi}^{n}-\frac{\Delta t}{\rho_{0}} \frac{\partial}{\partial \xi} p^{n} \\
\frac{\partial}{\partial \xi} u_{\xi}^{n+1} & =\mathbb{F}^{-1}\left\{i k_{\xi} \kappa \mathbb{F}\left\{u_{\xi}^{n+1}\right\}\right\}, \\
\rho_{\xi}^{n+1} & =\rho_{\xi}^{n}-\Delta t \rho_{0} \frac{\partial}{\partial \xi} u_{\xi}^{n+1}+\frac{\left(p^{n}\right)^{2}-\left(p^{n-1}\right)^{2}}{N \rho_{0} c_{0}^{4}} .
\end{aligned}
$$

Here $i$ is the imaginary unit, $k_{\xi}$ is the wavenumber in the $\xi$ direction, $\kappa$ is the $k$-space adjustment where $\kappa=\operatorname{sinc}\left(c_{0} k \Delta t / 2\right), \mathbb{F}$ and $\mathbb{F}^{-1}$ denote the forward and inverse Fourier transform, $\Delta t$ is the time step, and (5a)- $(5 \mathrm{~d}$ ) are repeated for each Cartesian direction in $\mathbb{R}^{N}$ where $\xi=(x)$ in $\mathbb{R}^{1}, \xi=(x, y)$ in $\mathbb{R}^{2}$, and $\xi=(x, y, z)$ in $\mathbb{R}^{3}$. The corresponding equation of state in discrete form is

$$
p^{n+1}=c_{0}^{2}\left(\rho^{n+1}+\frac{B}{2 A} \frac{1}{\rho_{0}}\left(\rho^{n+1}\right)^{2}-\mathrm{L}\right),
$$

where the total density is given by $\rho=\sum_{\xi} \rho_{\xi}$ and the discrete loss term is

$$
\mathrm{L}=-\tau \mathbb{F}^{-1}\left\{(k)^{y-2} \mathbb{F}\left\{\rho_{0} \sum_{\xi} \frac{\partial}{\partial \xi} u_{\xi}^{n+1}\right\}\right\}+\eta \mathbb{F}^{-1}\left\{(k)^{y-1} \mathbb{F}\left\{\rho^{n+1}\right\}\right\} .
$$


The discrete equations in (5) are iteratively solved using a time step based on the Courant-Friedrichs-Lewy (CFL) number, where $\Delta t=\mathrm{CFL} \Delta x / c_{\max }$. A CFL number of 0.3 typically provides a good balance between accuracy and computational speed for weakly heterogeneous media [10. At each time step, a velocity (or pressure) source can be included by adding the source values to the appropriate voxels within the computational domain. Similarly, the output from the simulation can be obtained by recording the acoustic variables at each time step at particular voxels within the grid. For the simulations presented here, the computational grids were also spatially and temporally staggered to improve accuracy, and an absorbing boundary layer included to prevent waves from wrapping around the domain [12. The codes were written as an extension to the k-Wave MATLAB toolbox (http://www.k-wave.org) and parallelization was achieved by interfacing with the GPU using Accelereyes Jacket. The reported simulation times correspond to execution on an NVIDIA TESLA C2070 which has 448 CUDA cores and 6 GB of onboard memory.

To validate the discrete equations, the one-dimensional propagation of a 5 $\mathrm{MPa}$ sinusoidal pressure source through a lossy medium with $\alpha_{0}=0.25 \mathrm{~dB}$ $\mathrm{MHz}^{-2} \mathrm{~cm}^{-1}$ was compared to the analytical expansion provided by Mendousse [5. The acoustic pressure versus the non-dimensional shock parameter $\sigma$ for $0 \leq \sigma \leq 3$ is shown in Fig. 11. There is a close agreement between the two solutions illustrating that cumulative nonlinear effects are correctly encapsulated.

\section{Harmonic Ultrasound Simulations}

To illustrate the applicability of the developed nonlinear $k$-space model to ultrasound simulation, the beam pattern produced by a linear phased array transducer was investigated. The utilized transducer model was $30 \mathrm{~mm}$ wide with 72 rectangular elements $5 \mathrm{~mm}$ in length and a kerf width of $0.25 \mathrm{~mm}$. The computational grid including the absorbing boundary layer was $120 \times 60 \times 30$ $\mathrm{mm}(512 \times 256 \times 128$ voxels $)$ supporting a maximum frequency of $3.54 \mathrm{MHz}$. The transducer was driven by a 5 cycle tone burst with a center frequency of 1 $\mathrm{MHz}$ and an equivalent single element source pressure of $1 \mathrm{MPa}$. The beam was electronically focused at $50 \mathrm{~mm}$ (with no elevation focusing), all elements were active, and no transmit apodization was used. The simulation was completed in 12.3 minutes (2360 time steps). The resulting beam patterns are shown using a linear plot scale in Fig. 2. These appear visually similar to those produced in $2 \mathrm{D}$ by Wojcik using the pseudospectral method 15 . Note, the same simulation performed using a $256 \times 128 \times 64$ voxel grid and 1080 time steps was computed in 51 seconds which demonstrates the excellent scaling characteristics of the model.

As a second example, the same transducer was used to simulate a B-mode ultrasound image of a scattering phantom (see Fig. 31). The ultrasound image was formed from 33 scan lines swept from $-32^{\circ}$ to $32^{\circ}$ using the conventional procedure of transmit and receive beamforming, time gain compensation, envelope detection, log compression, and scan conversion [9]. The total computational grid was $60 \times 60 \times 30 \mathrm{~mm}(256 \times 256 \times 128$ voxels $)$ supporting a maximum 


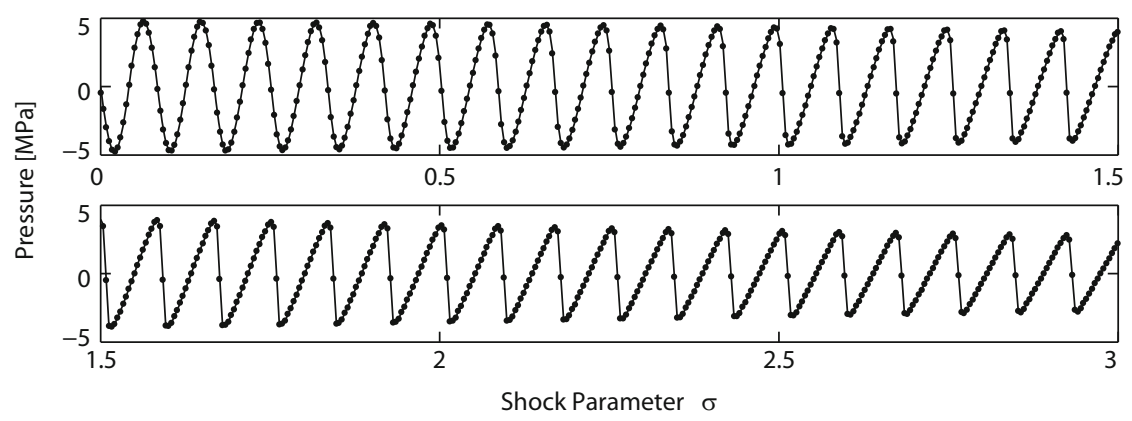

Fig. 1. Evolution of a finite amplitude pressure field in a one-dimensional medium with the shock parameter $\sigma$. The $k$-space solution is shown with a dotted line and the analytical expansion given by Mendousse is shown as a solid line for comparison.
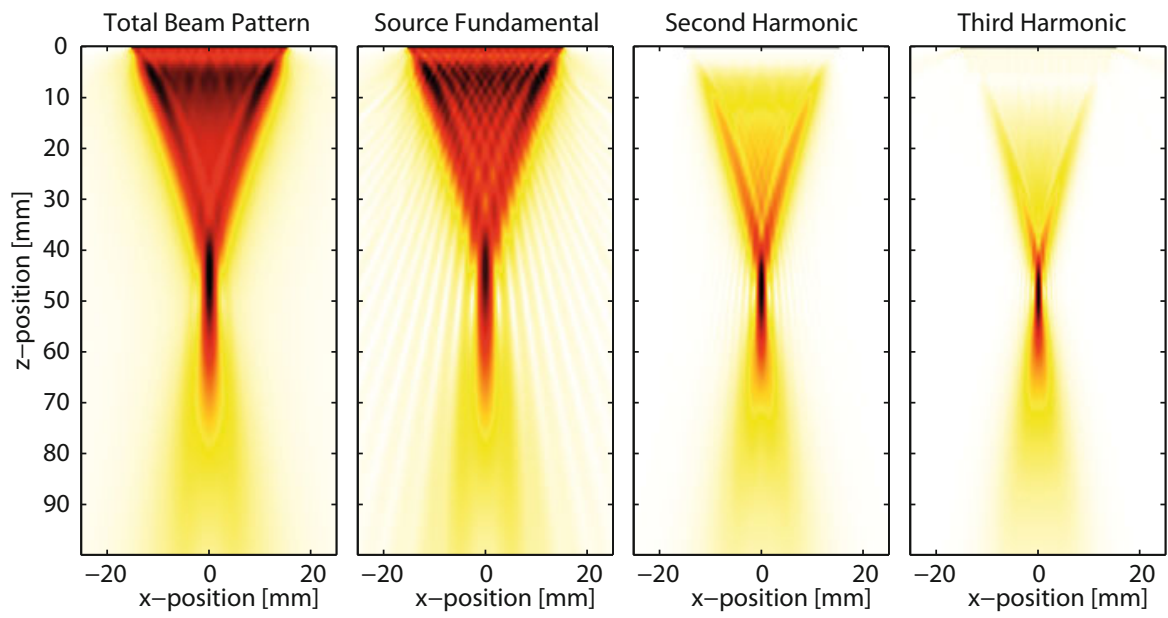

Fig. 2. Normalized beam patterns generated by a linear phased array transducer electronically focused at $50 \mathrm{~mm}$. The total beam pattern is extracted from the integrated frequency spectrum at each position, while the beam pattern at the fundamental and harmonics correspond to the relative spectral amplitudes at these frequencies.

frequency of $3.54 \mathrm{MHz}$ with a time step of $40 \mathrm{~ns}$. The transducer focus was set to $30 \mathrm{~mm}$ and the harmonic image was generated using phase inversion. The phantom was created by modulating the mean sound speed and density at each voxel using random Gaussian noise. For contrast, three spherical regions with increased scattering and impedance were defined. Each ultrasound scan line was computed in 4.78 minutes (corresponding to 1700 time steps).

In comparison, Daoud et al. reported simulation times of 2.86 hours per scan line for the same number of total voxels using a linear $k$-space model on a computer cluster with 2 nodes per scan line and 10 scan lines computed in parallel 4. While the relative computation time per scan line can be reduced by using 

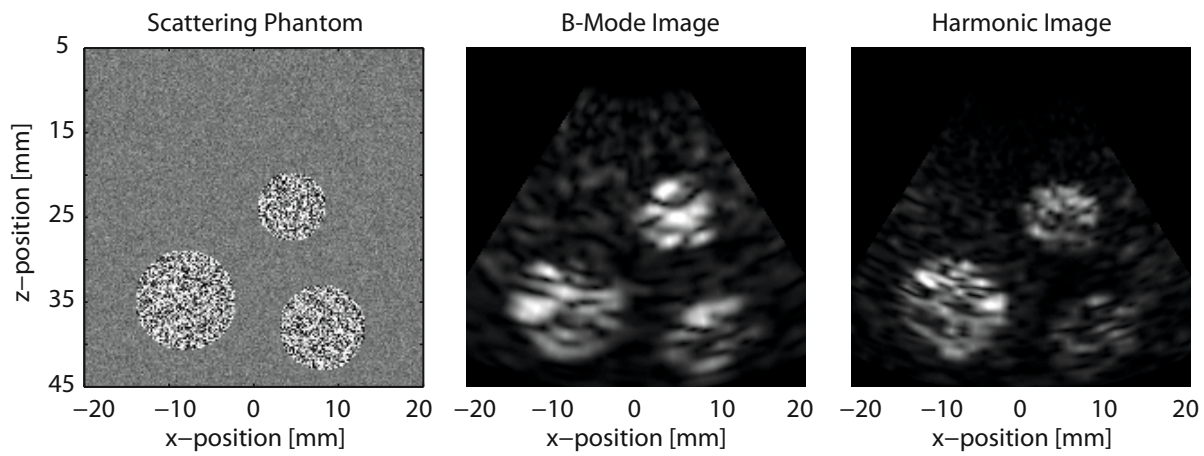

Fig. 3. Simulated B-mode ultrasound images of a scattering phantom. The images look realistic and contain the features and artifacts seen in B-mode images from commercial diagnostic scanners.

more nodes, this also reduces the computational efficiency due to the additional communication required (for 30 nodes the reported efficiency was approximately $90 \%$ and for 60 nodes $75 \%$ ). Thus, to achieve a comparable execution time to that reported here, nearly 100 nodes are required. This illustrates the architectural advantages of using GPUs for spectral methods over conventional computer clusters (the parallel performance of the FFT is discussed in more detail in [1]]). It is useful to note that for comparable simulations, finite difference methods yield significantly better performance when normalized by the number of voxels and time steps [87]. For example, Karamalis et al. reported simulation times on the order of 20 seconds per scan line for $2 \mathrm{D}$ simulations with $2^{22}$ grid elements and 6000 time steps using a finite difference method accelerated by the GPU 7 . However, for the same accuracy, the finite difference method requires as many as two orders of magnitude more voxels (in 3D) and an order of magnitude more time steps, thus the overall advantage of the $k$-space method is retained.

\section{Summary and Discussion}

A fast method for 3D simulations of nonlinear ultrasound propagation in biological tissue has been presented. This incorporates a novel equation of state that includes both nonlinearity and power law acoustic absorption. The spectral calculation of the spatial derivatives significantly reduces the requirement for dense computational domains compared to finite difference and finite element models. The model is applicable to all areas of ultrasound simulation but has particular relevance in treatment planning and exposure limit studies in which an accurate model of acoustic absorption is critical. The model is parallelized using a GPU which yields computational times significantly less than equivalent studies previously reported in the literature. This approach also has the potential to substantially reduce the total time taken to simulate ultrasound images by distributing scan lines across a cluster of GPUs. 
Acknowledgments. This work was supported in part by the Australian Research Council/Microsoft Linkage Project LP100100588 and the Engineering and Physical Sciences Research Council, UK. The authors would like to acknowledge Ramtin Shams and Jiř́ Jaroš for useful discussion, and the NVIDIA Academic Partnership Program for providing the TESLA C2070.

\section{References}

1. Aanonsen, S.I., Barkve, T., Tjotta, J.N., Tjotta, S.: Distortion and harmonic generation in the nearfield of a finite amplitude sound beam. J. Acoust. Soc. Am. 75(3), 749-768 (1984)

2. Baker, A.C., Berg, A.M., Sahin, A., Tjotta, J.N.: The nonlinear pressure field of plane, rectangular apertures: Experimental and theoretical results. J. Acoust. Soc. Am. 97(6), 3510-3517 (1995)

3. Cox, B.T., Kara, S., Arridge, S.R., Beard, P.C.: k-space propagation models for acoustically heterogeneous media: Application to biomedical photoacoustics. J. Acoust. Soc. Am. 121(6), 3453-3464 (2007)

4. Daoud, M.I., Lacefield, J.C.: Distributed three-dimensional simulation of B-mode ultrasound imaging using a first-order k-space method. Phys. Med. Biol. 54(17), 5173-5192 (2009)

5. Hamilton, M.F., Blackstock, D.T. (eds.): Nonlinear Acoustics. Acoustical Society of America, Melville (2008)

6. Jensen, J.A., Svendsen, N.B.: Calculation of pressure fields from arbitrarily shaped, apodized, and excited ultrasound transducers. IEEE Trans. Ultrason. Ferroelectr. Freq. Control 39(2), 262-267 (1992)

7. Karamalis, A., Wein, W., Navab, N.: Fast Ultrasound Image Simulation Using the Westervelt Equation. In: Jiang, T., Navab, N., Pluim, J.P.W., Viergever, M.A. (eds.) MICCAI 2010. LNCS, vol. 6361, pp. 243-250. Springer, Heidelberg (2010)

8. Pinton, G.F., Dahl, J., Rosenzweig, S., Trahey, G.E.: A heterogeneous nonlinear attenuating full-wave model of ultrasound. IEEE Trans. Ultrason. Ferroelectr. Freq. Control 56(3), 474-488 (2009)

9. Szabo, T.L.: Diagnostic Ultrasound Imaging. Elsevier, Burlington (2004)

10. Tabei, M., Mast, T.D., Waag, R.C.: A k-space method for coupled first-order acoustic propagation equations. J. Acoust. Soc. Am. 111(1), 53-63 (2002)

11. Tillett, J.C., Daoud, M.I., Lacefield, J.C., Waag, R.C.: A k-space method for acoustic propagation using coupled first-order equations in three dimensions. J. Acoust. Soc. Am. 126(3), 1231-1244 (2009)

12. Treeby, B.E., Cox, B.T.: k-Wave: MATLAB toolbox for the simulation and reconstruction of photoacoustic wave fields. J. Biomed. Opt. 15(2), 021314 (2010)

13. Treeby, B.E., Cox, B.T.: Modeling power law absorption and dispersion for acoustic propagation using the fractional Laplacian. J. Acoust. Soc. Am. 127(5), 2741-2748 (2010)

14. Wein, W., Brunke, S., Khamene, A., Callstrom, M.R., Navab, N.: Automatic CTultrasound registration for diagnostic imaging and image-guided intervention. Med. Image Anal. 12(5), 577-585 (2008)

15. Wojcik, G.L., Mould, J., Ayter, S., Carcione, L.M.: A study of second harmonic generation by focused medical transducer pulses. In: IEEE Ultrasonics Symposium Proceedings (Cat. No. 98CH36102), pp. 1583-1588 (1998) 